\title{
Characteristics of in-patients without psychosis
}

\author{
Implications for service provision and the recognition \\ of personality disorder
}

\author{
J. H. Dowson, P. Sussams and A. T. Grounds
}

\begin{abstract}
In a study of a population over 13 months, ratings were made from medical records of adults who were admitted to short-stay, non-secure (acute) psychiatric beds. Of 197 patients, 126 fulfilled DSM-IV criteria for specified psychoses; 57 of the 71 remaining nonpsychotic patients had a history of repeated self-harm, or threats of self-harm, and/or a substance-related disorder. Both these behaviours can be a manifestation $\alpha$, and be associated with, personclity disorder. It was considered that personality disorder made an important contritbution to the psychopathology in the nonpoychofic patients. The findings suggest a need to evaluate the altemative to in-patient care in a standard acute unit for such patients.
\end{abstract}

The objective of the present study was to investigate characteristics of in-patients without psychosis, to consider the role of personality disorder in such patients, and to assist the planning of services. The management of some non-psychotic patients in standard in-patient units is often problematic because of aggressive and other anti-social behaviour, which can be manifestations of personality disorder. 'Psychoses' are currently defined as specified DSMIV axis I disorders which can lead to severe incapacity and are often associated with delusions and/or hallucinations. The inadequate availability of psychiatric in-patient facilities in the UK invites an evaluation of the use of relatively expensive and scarce in-patient care.

A personality disorder consists of maladaptive patterns of motivated behaviour that are usually evident over a period of at least several years and are not considered to be part of other mental disorders (Dowson, 1995a). Patients with personality disorders are associated with many demands on health care provision, including inpatient care (Merikagas \& Weissman, 1986).
Adult psychiatric acute admissions to relatively short-stay, non-secure beds, from a part of the Cambridge Health District, were studied during a 13-month period. Patients were identified with or without a history of the following DSM-IV psychoses: schizophrenia and other psychotic disorders (except a history of psychotic disorder due to a general medical condition or substance-induced psychotic disorder, which did not exclude the patient from the nonpsychotic group): bipolar disorders; and depressive disorders with psychotic features (with delusions and/or hallucinations).

\section{The study}

In-patients of a psychiatric service for adults aged 16-65, from a geographically defined sector with a UK urban population of 52000 , were prospectively studied during a 13-month period. These patients included those of no fixed abode who were referred to the service from within the sector. The majority of patients were admitted to a 22-bed acute unit which catered for the range of disorders. Also, a minority of patients were admitted to specialised acute in-patient facilities for detoxification in relation to substance-related disorders or for the management of eating disorders.

Medical records provided the data for clinical ratings, based on DSM-IV, in relation to specified mental disorders. Consensus ratings were made by two of the authors, an experienced psychiatrist and an experienced graduate research assistant. The ratings were applied to each patient's adult lifetime, from the age of 16 to the end of the index admission or, if the index admission exceeded 26 weeks, ratings were applied to the period up to 26 weeks after the start of the index admission. (The index 
admission for each patient was the first period of in-patient management in an acute bed, at least part of which occurred during the study period of 13 months.)

In relation to the evaluation of self-harm, 'recurrent' was defined as two or more episodes. However, more than one incident during any 24hour period was considered to be just one episode.

Statistical analyses were carried out with the SPSS/PC programme (Norusis, 1988; Dowson, 1992) using a significance level of $\leqslant 0.05$. Chisquare analyses were applied to categorical variables, except where expected values were $\leqslant 5$, when the Fisher exact test was used.

\section{Findings}

The sector population consisted of 52000 residents of part of the City of Cambridge. UK of whom 35000 were between the ages of 16 and 65 . The sector populations in various age bands according to the 1991 National Census Profile were: $6 \%$ (15-19), 9.9\% (20-24), $24.5 \%$ (25-39), $21.2 \%(40-59)$ and $4.8 \%(60-64)$. The ethnic group was classified in the Census as 'White' for $94.5 \%$ of all sector residents.

During the study one patient was transferred from the acute unit to a medium-stay unit, and the index admission was considered to continue until discharge from in-patient care. Another patient was admitted to a secure unit soon after discharge from the index admission, and within 26 weeks of the start of the index admission; for this patient, the time in the secure unit was counted towards the number of in-patient days within 26 weeks of the start of the index admission.

The records of 197 patients were studied. A group of 126 patients fulfilled DSM-IV criteria for the psychoses defined as specified DSM-IV axis I disorders, and the remaining 71 patients formed the non-psychotic group; the mean ages for the groups were 34.7 (s.e.m 1.0) and 33.2 years (s.e.m 1.1) respectively.

Table 1 shows various characteristics of the groups, in particular the association of the nonpsychotic group with repeated self-harm, substance-related disorder and imprisonment. Fifty-seven of the 71 non-psychotic patients had a history of repeated self-harm (or threats of self-harm), a substance-related disorder, or both. Numbers of patients in these categories were 19,23 and 15 respectively. A smaller proportion of the psychotic group $(23.8 \%)$ had a history of criminality with police involvement compared with $36.6 \%$ of the non-psychotic group, while the corresponding figures for a history of imprisonment were 4 and $16.9 \%$ $(P \leqslant 0.05)$. Of the psychotic group $30.2 \%$ had at least one psychiatric admission during the two years before the start of the index admission, while the corresponding figure for the nonpsychotic group was $33.8 \%$. Of the psychotic group $23.8 \%$ were readmitted during the 26 weeks after the start of the index admission compared with $14.1 \%$ of the non-psychotic group. Nine of the psychotic group and three of the non-psychotic group were long-term wandering homeless. The mean length of the index admission (days) for patients discharged within 26 weeks was $41.2(n=121)$ for the psychotic group and $23.7(n=70)$ for the non-psychotic group.

Sixty-four of the 71 non-psychotic patients had at least one DSM-IV axis I mental disorder, other than a substance-related disorder, within the 12 weeks prior to the start of the index admission. These involved mood disorder (mainly a depressive disorder) in 61 patients, of which 28 also had a substance-related disorder, while 13 had a recent history of an axis I disorder other than a mood disorder or a substance-related disorder.

\section{Comment}

Many studies have noted a high prevalence of personality disorder among in-patients with a variety of axis I mental disorders (Cutting et al, 1986 ) and in the present study, $80 \%$ of the nonpsychotic patients had a history of repeated self-harm and/or a substance-related disorder, both of which are commonly associated with personality disorder (Nace et al, 1991; Russ, 1992). Also, both repeated self-harm and features of DSM-IV substance-related disorders (including substance misuse) can be manifestations of personality disorder in many patients. This is because repeated self-harm may be a feature of borderline personality disorder, while some substance-related disorders can be considered as examples of maladaptive impulse control, specified as a feature of personality disorder in DSM-IV's general diagnostic criteria for a personality disorder. We conclude that personality disorder made a considerable contribution to the psychopathology in the nonpsychotic patients. The incidence of personality disorder in in-patient samples may often be underestimated if priority is given to co-occurring axis I disorders, in particular mood disorders and substance-related disorders (Dowson, 1995b).

It is likely that the present findings, for a service which has frequent unavailability of admission beds, reflect service patterns in similar urban areas.

Many non-psychotic in-patients present management problems because of substance mis- 
Table 1. Characteristics of 197 patients aged 16-65 who received in-patient psychiatric management during a 13-month period

\begin{tabular}{|c|c|c|c|}
\hline & $\begin{array}{l}\text { Psychotic } \\
(n=126)\end{array}$ & $\begin{array}{l}\text { Non-psychofic } \\
(n=71)\end{array}$ & Significance \\
\hline \multicolumn{4}{|l|}{ Gender } \\
\hline Male & 65 & 45 & \\
\hline Female & 61 & 26 & \\
\hline In-patient days during study period' & 7766 (76\% of total) & 2450 ( $24 \%$ of total) & \\
\hline $\begin{array}{l}\text { Mean of in-patient days/patient during } \\
26 \text { weeks after start of index admission } 2\end{array}$ & 56.0 (s.e.m 4.4) & $28.3($ s.e.m 4.6) & $t=4.1^{*}$ \\
\hline $\begin{array}{l}\text { Compulsory detention during index admission } \\
\text { History of repeated self-harm behaviour }\end{array}$ & $57(45.2 \%)$ & $10(14.1 \%)$ & $x^{2}=19.6^{*}$ \\
\hline Any & $12(9.5 \%)$ & $34(47.8 \%)$ & $\chi^{2}=37.3^{*}$ \\
\hline Overdose & $12(9.5 \%)$ & $23(32.4 \%)$ & $\chi^{2}=16.3^{*}$ \\
\hline Self-mutilation & $12(9.5 \%)$ & $11(15.5 \%)$ & NS \\
\hline Self-harm (other) & $1(0.8 \%)$ & $9(12.7 \%)$ & (Fisher)* \\
\hline Threats of self-harm & $7(5.6 \%)$ & $11(15.5 \%)$ & $\chi^{2}=5.4^{*}$ \\
\hline \multicolumn{4}{|l|}{ History of substance-related disorder } \\
\hline Any & $30(23.8 \%)$ & $38(53.5 \%)$ & $\chi^{2}=17.7^{*}$ \\
\hline Alcohol misuse & $15(11.9 \%)$ & $32(45.1 \%)$ & $\tilde{\chi}^{2}=27.5^{*}$ \\
\hline Illicit drug misuse & $18(14.3 \%)$ & $18(23.3 \%)$ & $\tilde{\chi}^{2}=3.7^{*}$ \\
\hline IV Drug use & $3(2.4 \%)$ & $5(7.0 \%)$ & NS \\
\hline \multicolumn{4}{|l|}{ Transterred to start of index admission from } \\
\hline General hospital facilities & $15(11.9 \%)$ & $18(25.4 \%)$ & $\chi^{2}=5.9^{*}$ \\
\hline Police custody & $14(11.1 \%)$ & $7(9.9 \%)$ & NS \\
\hline \multicolumn{4}{|l|}{ Accommodation prior to index admission } \\
\hline No flxed abode ${ }^{3}$ & $11(8.7 \%)$ & $8(11.3 \%)$ & NS \\
\hline Hostel & $41(32.5 \%)$ & $8(11.3 \%)$ & $\chi^{2}=11.0^{*}$ \\
\hline Rented housing & $53(42.1 \%)$ & $48(67.6 \%)$ & $\chi^{2}=11.8^{*}$ \\
\hline Other & $21(16.7 \%)$ & 7 (9.9\%) & NS \\
\hline
\end{tabular}

1. In-patient days in relation to acute (i.e. short-stay, non-secure) in-patient facilities; these did not include the inpatient care for two patients who received medium-stay or secure in-patient facilities during the study period.

2. In-patient days which included admissions of two patients to medium-stay or secure in-patient facilities.

3. The following criteria were met in relation to the 28 days before index admission: had 'slept rough' at least one night and/or had moved accommodation at least once; and future accommodation was uncertain at start of index admission.

$* P \leqslant 0.05$

use and/or frequent aggressive behaviour which can adversely affect the care provided for other patients. Various alternatives to a standard acute in-patient unit for such patients have included an 8-bed brief-stay unit for the range of psychiatric emergencies, including those involving patients for whom personality disorder is the most apparent diagnosis (Brestow et al, 1993). Another arrangement, which was provided for patients selected on the basis of personality disorder, also involved a structured time-limited in-patient programme (Silk et al, 1994). This regime was part of an otherwise standard psychiatric unit which accepted referrals of compliant patients from other units. Also, complementary or alternative provision for patients with personality disorder may involve medium- or long-term support in a range of out-patient, day-care, hostel and hospital-hostel facilities (Linehan et al, 1993;
Kurian et al. 1994). Medium- or long-term individual supportive psychotherapy can be particularly important for patients with borderline personality disorder.

The findings show that patients admitted with non-psychotic disorders have a high incidence of repeated self-harm and substance-related disorders: it is considered likely that personality disorder made an important contribution to their psychopathology and that these patients make significant demands on in-patient psychiatric services in the UK. There is a need to evaluate a range of alternatives to their in-patient care in a standard acute unit.

\section{Acknowledgements}

This work was supported by a grant from the East Anglian Regional Health Authority. The authors thank Ms Lorraine Allen for her help. 


\section{References}

Breslow, R. E., KuINGer, B. I. \& ERICKSON, B. J. (1993) Crisis hospitalization on a psychiatric emergency service. General Hospital Psychiatry. 16. 307-315.

Cutting, J., Cowen, P. J.. MANN, A. H., et al (1986) Personality and psychosis: use of the standardised assessment of personality. Acta Psychiatrica Scandinavica, 73, 87-92.

Dowson, J. H. (1992) Associations between self-induced vomiting and personality disorder in patients with a history of anorexia nervosa. Acta Psychiatrica Scandinavica, 86, 399-404.

- (1995a) Personality disorders: basic concepts and clinical overview. In Personality Disorders: Recognition and Clinical Management leds J. H. Dowson \& A. T. Grounds). pp. 3-42. Cambridge: Cambridge University Press.

- (1995b) Personality disorders: less specific clinical presentations and epidemiology. In Personality Disorders: Recognition and Clinical Management (eds J. H. Dowson \& A. T. Grounds), pp. 128-158. Cambridge: Cambridge University Press.

Kurian, M.. George, S.. BALLARD, C. G.. et al (1994) Audit of new longstay patients in a district general hospital. Irish Joumal of Psychological Medicine, 11, 42-43.

LINEHAN. M. M., HEARD, H. L.. \& ARMSTRONG, H. E. (1993) Naturalistic follow-up of a behavioural treatment for chronically parasuicidal borderline patients. Archives of General Psychiatry. 60. 971-974.
Merikangas, K. R. \& Weisman, M. H. (1986) Epidemiology of DSM-III Axds II personality disorders. In APA Annual Revlew, vol. 5 (eds A. J. Frances \& R. E. Hales), pp. 258278. Washington. DC: American Psychiatric Press.

NACE, E. P.. DAVID, O. W. \& GASPAR, J. P. (1991) Axds II comorbidity in substance abusers. American Journal of Psychiatry. 148, 118-120.

NORUSIS. M. J. (1988) SPSS/PC ${ }^{+}$Advanced Statistics for the IBM PC/XT/AT and PS/2. Chicago. IL: SPSS.

Russ, M. T. (1992) Self-injurious behaviour in patients with borderline personality disorder: biological perspectives. Journal of Personality Disorders, 6, 64-81.

SILK, K. R., EISNER, W., ALLPORT, C., et al (1994) Focused time-limited inpatient treatment of borderline personality disorder. Joumal of Personality Disorders. 8. 268-278.

* Jonathan H. Dowson, Department of Psychiatry. Level E4, Addenbrooke's Hospital, Cambridge CB2 2Q9; Pamela Sussams and Adrian T. Grounds, Department of Psychiatry. University of Cambridge.

*Correspondence

\section{The Psychotherapy of Psychosis}

\section{Edited by Chris Mace and Frank Margison}

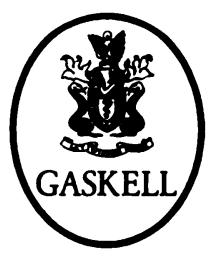

This book provides an unusually comprehensive survey of the current state and prospects of psychological methods of treatment for people with schizophrenia and other psychotic illnesses. It will be an invaluable resource for mental health professionals and clinical managers involved in their care, and essential reading for psychiatrists at all levels of experience.

The three traditions of psychotherapy and integrated approaches are covered. Recent research in the process and outcome of psychotherapy is reviewed and summarised. Clear advice is also given on treatment techniques and settings with reference to national policies. As with other titles in the series, there is frequent use of boxes, tables and figures to set out important points and key information. 1997, 296pp, ISBN $1901242048, £ 25.00$

Gaskell publications are available from Booksales, Publications Department,

Royal College of Psychiatrists, 17 Belgrave Square, London SW1X 8PG

$($ Tel. $+44(0) 1712352351$, extension 146). The latest information on College publications is available on the INTERNET at: http://www.demon.co.uk/rcpsych/ 